\title{
Algorithmic Methodology Based Automobile Theft Detection and Prevention System
}

\author{
Ms. Monika Soni \\ Department of Computer Engineering Bikaner Rajasthan India \\ monikasoni@gmail.com
}

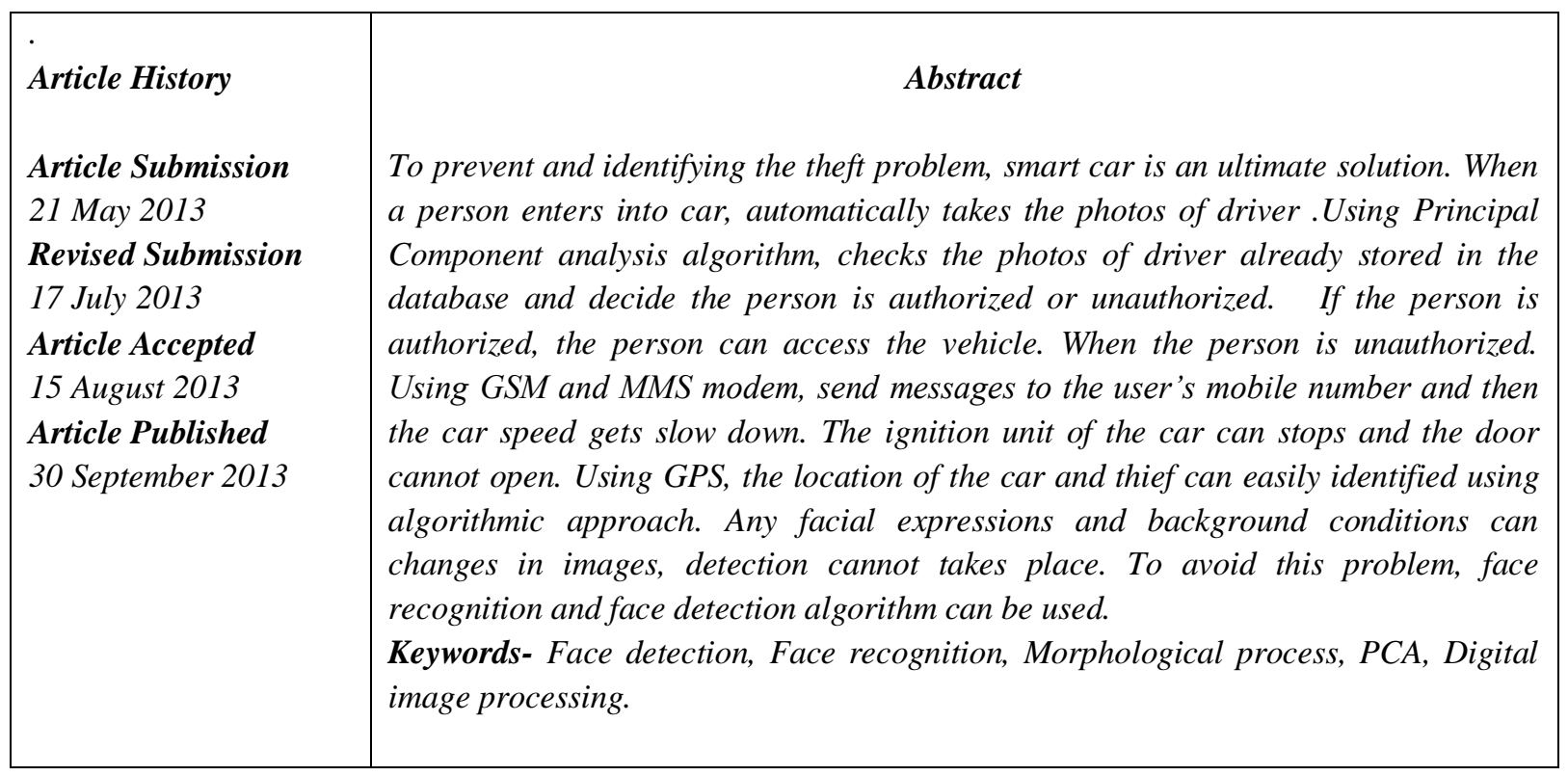

\section{Introduction}

The access oriented security control systems play a vital role in safety schemes through ICs, public keys and secret pins. Identification of humans characters by organization are nearly all contemporary and convoluted admittance be in command of sanctuary systems [1]. They are measured not succeed-secure, for the reason that it may be trouble-free to lacerate a secret words, straightforward to search out backside in a row entrenched on a path, easy to private and public data, but it is approximately unworkable to compose a model of distinguishing person distinctiveness [3]. There are poles apart measurable characteristics know-how like influence appreciation, mark gratitude, vitamin A aldehyde searching circular structure in eye credit, fiddle with feature identification. Measure up to supplementary identification of human modus operandi recompense of expression detection [2][3]. The Straight forward to position, can make use of presented figure confine plans (video camera, sanctuary camera etc. It does not necessitate client support nothing like touch produce. Citizens can be acknowledged devoid of their understanding. It is easier to achieve a snapshot fairly than a manipulate make or circular structure in eye check.

The face decision needs expression identification stage with a specified image. The face area is extracted through face recognition phase. Most of the entry management system comprises of face identification and face recognition which may prevent illegal car theft and provide alerting schemes. When driver enters car, a camera present in the façade system identifies the image of the driver and verify its authenticity [4].

\section{Related Works}

Many researchers have proposed numerous façade detection system algorithms that group the parts of the face. Façade detection system uses a dedicated video camera to ensure the face recognition. The shape matching algorithm proposed in earlier days helped to match and distinguish between face features [5][6]. To strengthen this module, expression detection algorithm was framed in which face near to the source is white and the 
remaining spots are black. This image is converted to Eigen values with respect to the saved face database. PCA algorithms are also used for face recognition where images are converted into principal components related to orthogonally principle [7]

PCA is followed by ordering algorithm which features lowering down of dimensions of the image which takes less remembrance time [8]. Image dispensation which is a type of image processing considers the input image as a two dimensional quantity. Imaging systems are boon to verification systems where the input image is converted into information to be fetched. The imaging systems find its significance in remote sensing, biometrics, computer automation, astronomy and defense sectors [7][9].

\section{Proposed Image Dispensation Based Vehicular Mugging Detection and Avoidance System}

This proposed arrangement contains hardware component unit amalgamated with software unit. Hardware unit contains image attainment system, network of sensors, GSM and relays. The image attainment is performed with a digitalized camera. The soft-ware unit does picture pre-processing through expression exposure technique and face appreciation techniques. The overall proposed block diagram is shown in fig.1.

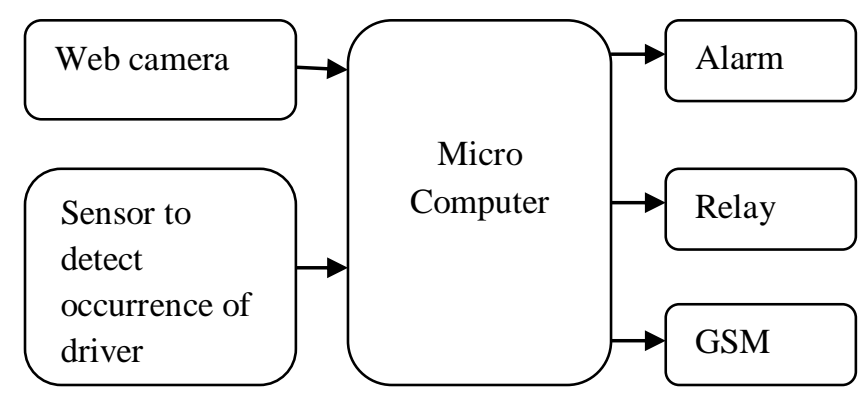

Fig. 1 Block diagram of the proposed system

The camera will capture the image of the driver and sends the signal to microprocessor unit once the PIR sensor is activated. The captured image is subjected to face detection and recognition phase leading to verification phase. If the image mismatch happens, information will sent to owner using GSM module. The structure of face identification system is exposed in figure 2.

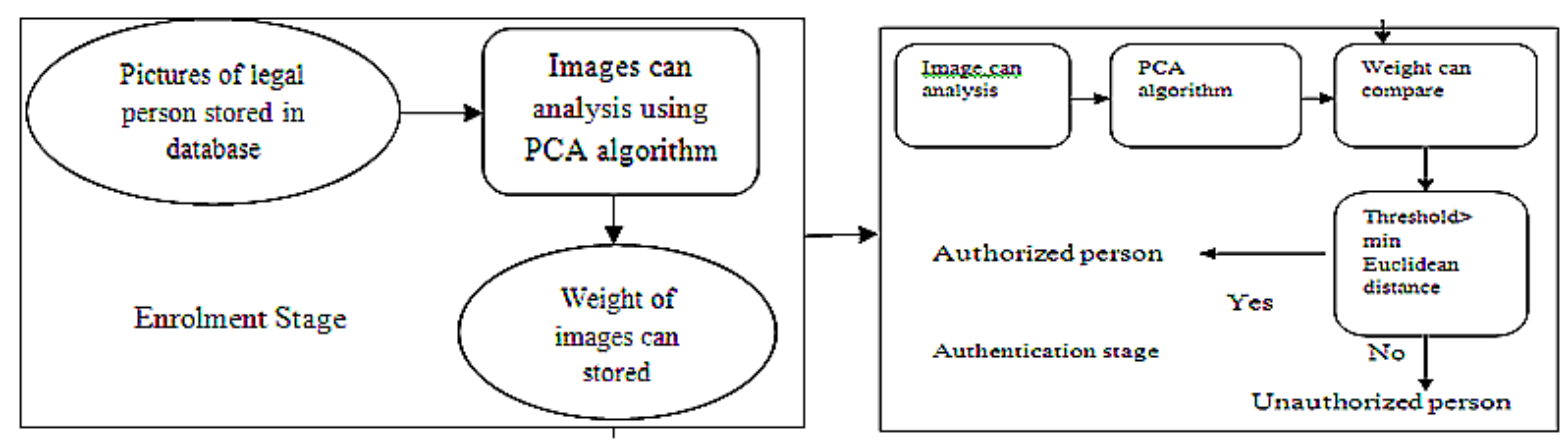

Fig.2. Block diagram of face recognition system

The hardware implementation of image dispensation based vehicular mugging detection and avoidance system is implemented through PIC16F877A microprocessor, ARM microcontroller, PIR sensor, MAX232, relay, switch circuits and GSM module. The structural diagram of hardware architecture is shown in Fig.3 


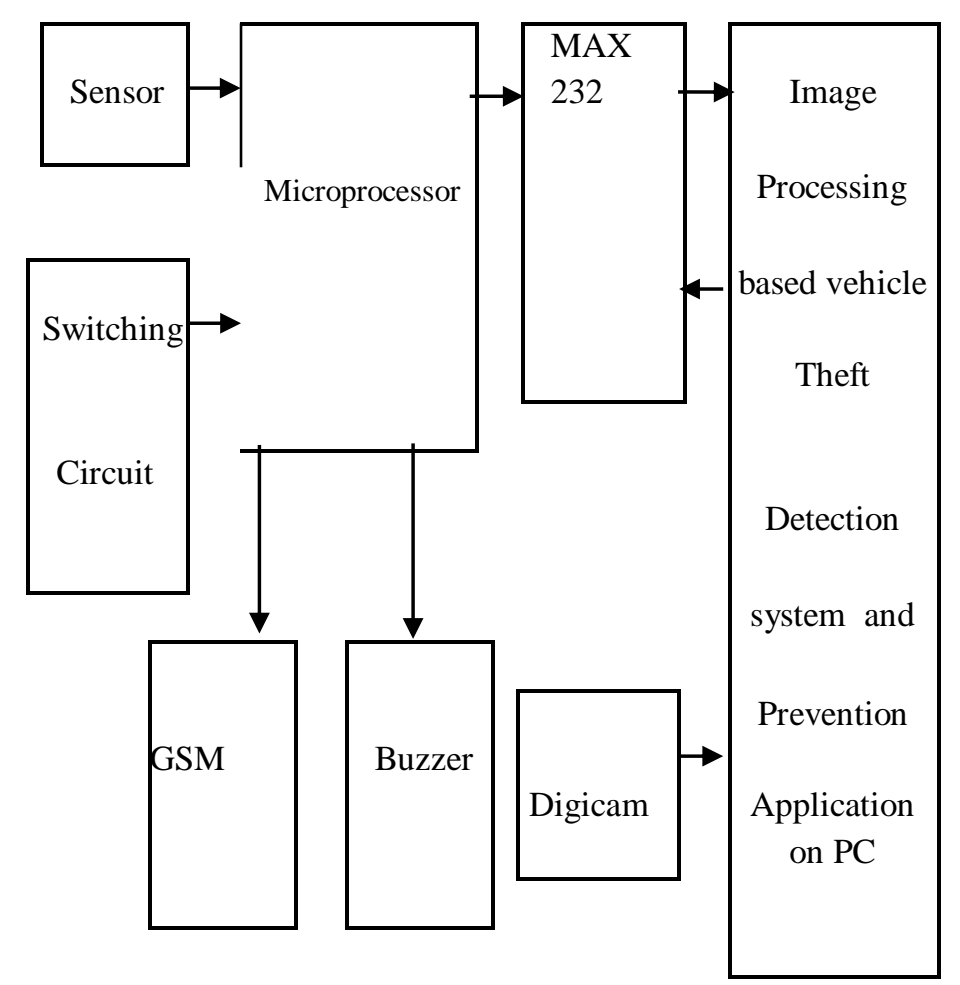

Fig. 3. Hardware block diagram

\section{Algorithm Simulation Results and Discussion}

Face detection is the initial step of the proposed system and simulated using MATLAB7.6.0 (R2008a). This helps in face extraction leading to identification of image parameters like skin color. The distance of the user plays a vital role as closer images of the skin will be mismatched as face.

In the proposed algorithm, color segmentation and morphological progression are introduced. The sectioned covering area at present assessment is high and mighty that the facade would consists of a negligible sum lone break as it has watch, ease and a jaws.

If individual separation is premeditated at a moment, $\mathrm{H}$ is high. When numeral of tear $>0$, conclude whether thickness/altitude of piece fabrication in the gap $0<$ percentage 4 . If fraction is content, the fraction is distinguish as facade, as well not a frontage. At this time we judge that holder requirements to verify two drivers. The fifteen set of images take by webcam is shown in figure 4 . 


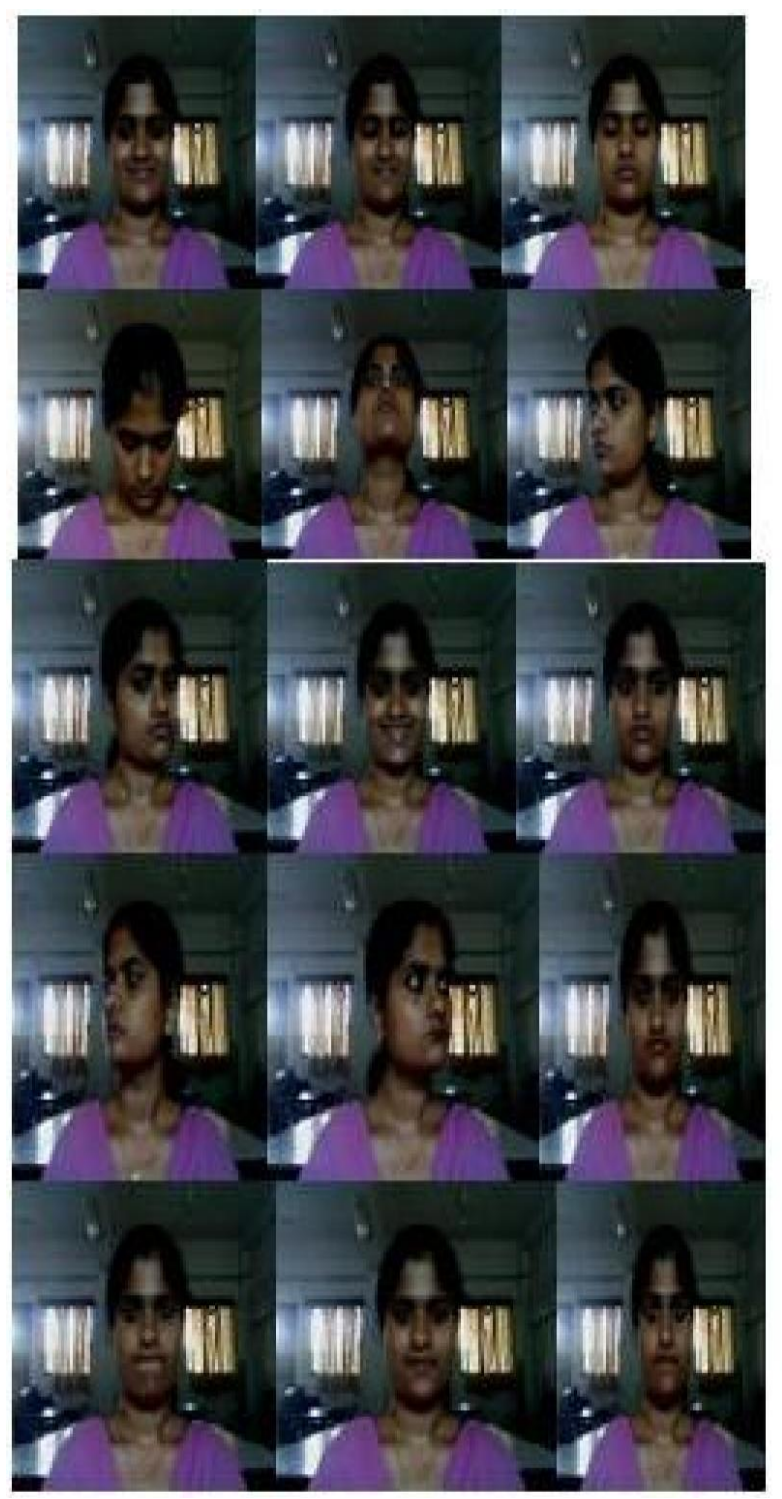

Fig. 4 Set of photos taken by webcam

The proposed algorithm for image extraction is shown below:

1. Acquire education locate of similes

Brink $=\mathrm{B}+\mathrm{M}$

2. Distinguish facade of all exercise descriptions

3. Perform DCT normalization for same clarification = square root (M1)

4. Enter these descriptions as instruction position into look respect step

5. Calculate load vector for participation guidance set by means of PCA algorithm

6. Provide fresh analysis illustration

7. Conclude whether given picture is legal or not legal by means of Principal Component Analysis algorithm

8. If person is legal, control on conveys is associated among explosion twist and sequence and thus provide admission to car.

9. If driver is not a legal person spread wont button and driver won't have admission to car. If he is not a legal person a sound can arises and a memo will be launch to holder's cellular phone saying that 'CAR IN CRITICAL CONDITION'. 
The extracted images using the above algorithm are shown in figure 5.

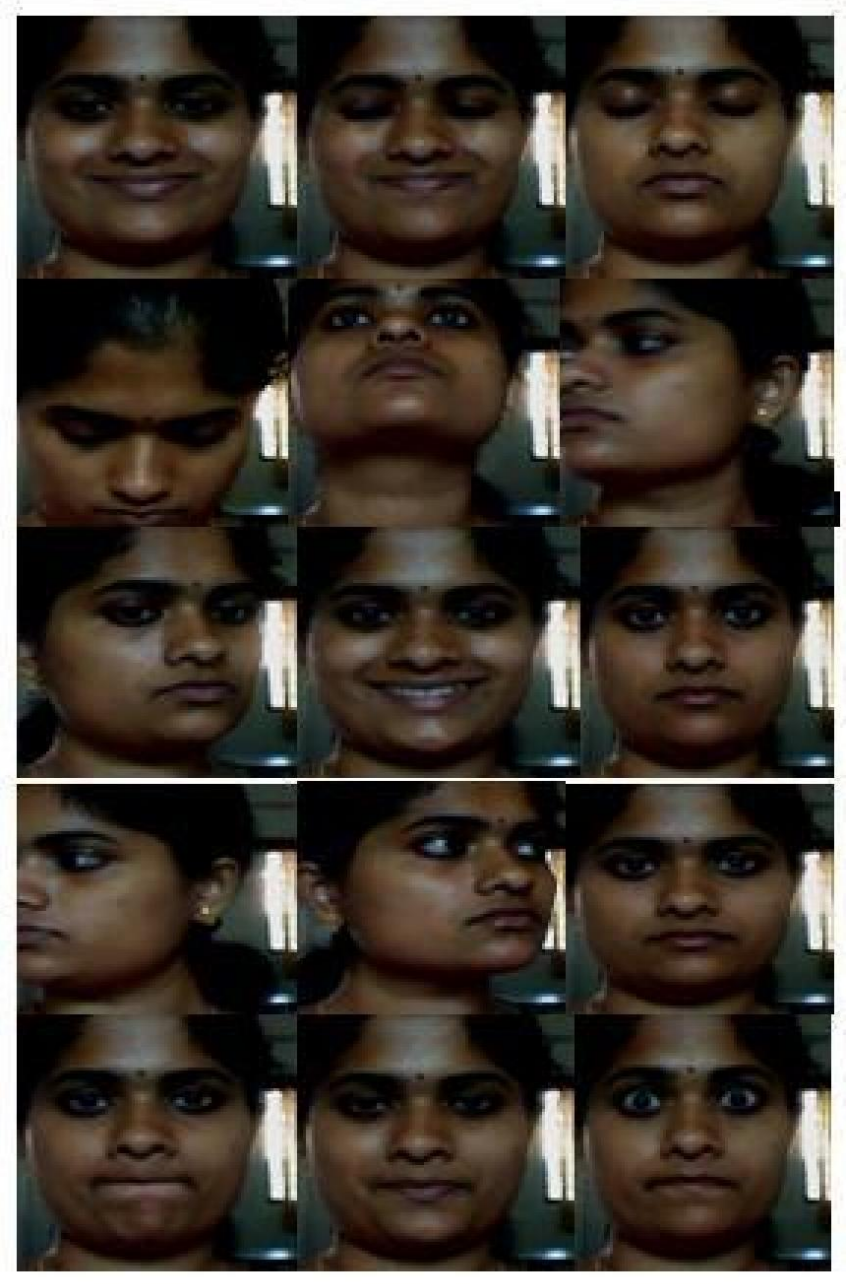

Fig.5 Set of Extracted faces using algorithmic approach

The picture processing based vehicular mugging detection and avoidance system is developed comprising of registered faces. If he is legitimate, switch on spread that is associated flanked by explosion coil and battery thereby providing contact to vehicle. If he is not rightful person, spread is switched off and hindered him from starting vehicle. The information is also passed to the owner mobile number.

\section{Results and Discussion}

Image processing based automobile vehicular mugging detection and avoidance system was developed using amalgamation of software and hardware. Using DCT normalization, face recognition is completed. Using the proposed threshold detection algorithm, two images with high accuracy are selected. The threshold determination is performed by using 15 images. This method avoids non-uniform classification of images leading to high efficiency. In this paper a system for vehicular mugging detection and avoidance system has been proposed using an algorithmic approach. The system helps to reduce augmented amount of automobile larceny. The system can be used in a range of applications similar to PC login security, robotics, presence marking system, activists screening and passport authentication. 


\section{References}

[1] Xiaofei Zhou, Yong Shi, Peng Zhang, Guangli Nie, Wenhan Jiang, "A New Classification Method for PCA-based Face Recognition," International Conference on Business Intelligence and Financial Engineering,2009.

[2] WeilongChen, MengJooEr, "Illumination Compensation and Normalization for Robust Face Recognition Using Discrete Cosine Transform in Logarithm Domain," IEEE Transactions on Systems, Man and Cybernetics, Vol.36, No.2, April 2006

[3] R. Kanjee, A. K. Bachoo and J. Carroll, "Vision-based Adaptive Cruise Control using pattern matching," 2013 6th Robotics and Mechatronics Conference (RobMech), Durban, 2013, pp. 93-98.

[4] Kresimir Delac and Mislav Grgic, "Face recognition", I-TECH Education and Publishing, Vienna, Austria, 2007

[5] M.Turk. and A.Pentland., "Face recognition using eigen faces", Proceedings of IEEE Conference on Computer Vision and Pattern Recognition, Maui, Hawaii, pp. 586-591, 3-6 June 1991.

[6] M. Turk, A. Pentland, "Eigenfaces for Recognition", Journal of Cognitive Neuroscience, Vol. 3, No. 1, pp. 71-86, 1991

[7] Dr. Arti Khaparde, Sowmya Reddy.Y Swetha Ravipudi ,"Face Detection Using Color Based Segmentation and Morphological Processing - A Case Study,"

[8] S. Sharma, "Embedded Systems -- A Security Paradigm for Pervasive Computing," 2013 International Conference on Communication Systems and Network Technologies, Gwalior, 2013, pp. 472-477.

[9] Studnia, V. Nicomette, E. Alata, Y. Deswarte, M. Kaâniche and Y. Laarouchi, "Survey on security threats and protection mechanisms in embedded automotive networks," 2013 43rd Annual IEEE/IFIP Conference on Dependable Systems and Networks Workshop (DSN-W), Budapest, 2013, pp. 1-12.

[10] J. Hu, J. Li and G. Li, "Automobile Anti-theft System Based on GSM and GPS Module," 2012 Fifth International Conference on Intelligent Networks and Intelligent Systems, Tianjin, 2012, pp. 199-201.

[11] Ahilan and E. A. K. James, "Design and implementation of real time car theft detection in FPGA," 2011 Third International Conference on Advanced Computing, Chennai, 2011, pp. 353-358.

[12] P. S. R. Teja, V. Kushal, A. S. Srikar and K. Srinivasan, "Photosensitive security system for theft detection and control using GSM technology," 2015 International Conference on Signal Processing and Communication Engineering Systems, Guntur, 2015, pp. 122-125. 